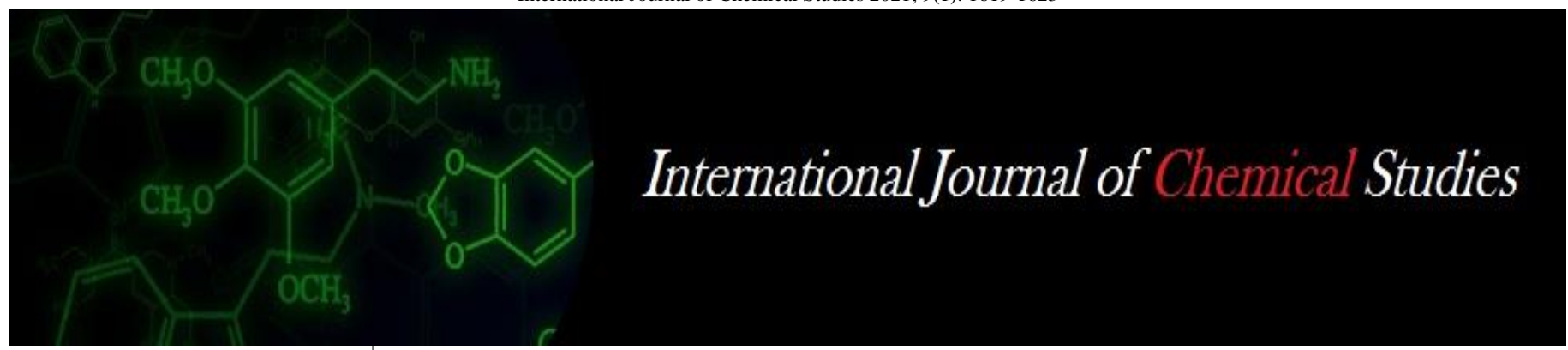

P-ISSN: 2349-8528

E-ISSN: 2321-4902

www.chemijournal.com

IJCS 2021; 9(1): 1619-1623

(C) 2021 IJCS

Received: 19-11-2020

Accepted: 21-12-2020

Namrata P Malge

M.Sc. Student, Department of

Genetics and Plant Breeding,

Faculty of Agriculture,

Dr. Panjabrao Deshmukh Krishi

Vidyapeeth, Akola,

Maharashtra, India

Dhaneshwari N Pachkhande M.Sc. Student, Department of Genetics and Plant Breeding.

Faculty of Agriculture,

Dr. Panjabrao Deshmukh Krishi

Vidyapeeth, Akola,

Maharashtra, India

Ugale KN,

M.Sc. Student, Department of Genetics and Plant Breeding.

Faculty of Agriculture,

Dr. Panjabrao Deshmukh Krishi

Vidyapeeth, Akola,

Maharashtra, India

Lande SS,

Assistant Professorr of Genetics and Plant Breeding, Pulses

Research Unit, Dr. PDKV

Akola, Maharashtra, India

Archana W Thorat

Senior Research Scientist, Pulses

Research Unit, Dr. PDKV,

Akola, Maharashtra, India

Corresponding Author:

Namrata P Malge

M.Sc. Student, Department of

Genetics and Plant Breeding,

Faculty of Agriculture, Dr.

Panjabrao Deshmukh Krishi

Vidyapeeth, Akola

Maharashtra, India

\section{Estimation of heterosis for yield and it's components in chickpea (Cicer arietinum $\mathrm{L}$.)}

\author{
Namrata P Malge, Dhaneshwari N Pachkhande, Ugale KN, Lande SS and \\ Archana W Thorat
}

DOI: https://doi.org/10.22271/chemi.2021.v9.i1w.11460

\begin{abstract}
Chickpea is highly self-pollinated crop and the scope for exploitation of hybrid vigour will depend on the direction and magnitude of heterosis, biological feasibility and the type of gene action involved. Also study of heterosis will have the direct bearing on the breeding methodology to be employed for varietal improvement. The experiment was conducted at Pulses Research Unit, Dr. P.D.K.V. Akola, during rabi season of 2018-19. In this study, four lines were crossed with six male parents by using line $\mathrm{x}$ tester design and twenty four hybrids were developed along with the variety JAKI-9218 as standard check have been evaluated in RBD with two replication. The highest mean performance was recorded in PDKV Kanchan x GJG-0814 (27.65g) and significant standard heterosis (15.45\% over check JAKI-9218), heterobeltiosis (20.22) and average heterosis (25.68). Another cross Phule Vikram x C-1821 revealed high mean performance for $(26.50 \mathrm{~g})$ for seed yield per plant also recoded high magnitude of useful heterosis (10.65 over check JAKI-9218).
\end{abstract}

Keywords: Heterosis, heterobeltiosis, standard heterosis and line $\mathrm{x}$ tester design chickpea

\section{Introduction}

Chickpea (Cicer arietinum L.) is the third most important food legume (after dry bean and pea) globally, grown in over 40 countries representing all the continents. Over $90 \%$ of area, production and consumptions are in developing countries. Presently, the most important chickpea producing countries are India, Australia, Pakistan, Turkey, Myanmar, Ethiopia, Iran USA, Canada and Mexico. India is the largest chickpea producer as well as consumer in the world sharing 69.75 and 70.71 per cent of the total area and production, respectively.

However, production and productivity of chickpea have been stagnant for the past three decades. Therefore there is need to incorporate drought tolerance into kabuli chickpeas for attaining stable yields under receding soil moisture conditions. Therefore, keeping this in mind the present investigation was carried out to estimate degree of heterosis. The exploitation of heterosis is one of the breeding strategies to enhance the productivity. Line $\mathrm{x}$ tester analysis is an extension of top cross method in which several testers are used which provides information about general and specific combining ability of parents and at the same time it is helpful in identifying best heterotic crosses.

\section{Method and Materials}

Present research was conducted at Pulses Research Unit, Dr. PDKV. Akola. The parent material for the study consisted of four lines viz., PDKV Kanchan, Phule Vikram, AKG-1303, Chanoli and six tester viz., GAU-1107, GJC-3, WR-315, JCP-101, C-1821 and GJG-0814. Crossing work was done in rabi 2018-19 and evaluation was done in rabi 2019-20, resultant 24 crosses ten parents and checks JAKI-9218 were sown in RBD design with two replications for evaluation. Each entry was sown in one row of $3 \mathrm{~m}$ length in each replication with inter and intra-row spacing was $45 \mathrm{~cm}$ and $10 \mathrm{~cm}$, respectively. All the standard agronomic and plant protection measures were used.

The data was recorded on plant basis and plot basis, from each genotype in each replication on 5 randomly selected plants and their average value was computed for ten quantitative traits viz., Plant stand, days to $50 \%$ flowering, days to maturity, plant height $(\mathrm{cm})$, number of primary branches, number of secondary branches, number of pods per plant, 100 seed weight 
( $\mathrm{g}$ ), seed yield per plant $(\mathrm{g})$, number of seeds per pod, number of empty pods per plant, growth habit, loddging resistance. Heterosis was calculated over mid parent, better parent and standard checks for seed yield and other yield contributing traits. Data in each experiment of all entries was subjected to analysis of variance (Panse and Sukhatme, 1967) ${ }^{[7]}$ for testing the significance of treatments.

\section{Result and Discussion}

The mean sum of square form analysis of variances of ten chickpea traits are presented in (Table 1). It is evident for the table that highly significant differences among chickpea genotypes, parent, parent vs crosses. Highly significant crosses among parent vs crosses indicated the presence of considerable heterosis for all the studied traits. The significance differences could be attributed to genetically diverse nature of female and male parents. The estimates of heterosis, heterobeltiosis and standard heterosis for indicated chickpea traits are presented in (Table 2). Days taken to 50\% flowering are of significance in the since that if the flowering starts earlier, sufficient time will be available for grain formation process. Thus early flowering is desirable in chickpea and negative heterosis for days to $50 \%$ flowering is useful. Out of 24 crosses, 1 and 3 crosses showed significant and negative average heterosis and heterobeltiosis respectively. Highest significant negative average heterosis and heterobeltiosis was observed in the cross PDKV Kanchan $\times$ GAU-1107 (-16.59\%). The cross PDKV Kanchan $\times$ GAU$1107(-18.52 \%)$ exhibited highest negative heterobeltiosis. The cross Phule Vikram $\mathrm{x}$ GJC-3 recorded significant negative standard heterosis over check JAKI-9218. Means these crosses are early flowering. These results are in close conformite with the finding of Singh and Paroda (1989) ${ }^{[5]}$, Kulkarni et al. (2004) ${ }^{[3]}$ who also reported negative heterosis for days to $50 \%$ flowering. Days taken to maturity are an effective trait for earliness and thus negative heterosis for this trait is desirable. Out of 24 crosses 8, 10 and 4 crosses showed significant, negative and highest average heterosis, heterobeltiosis and standard heterosis respectively. Maximum significant and negative average heterosis was observed in cross Phule Vikram x GAU-1107 (-3.09\%). Highest significant and negative heterobeltiosis was observed in cross Phule Vikram $\times$ GAU-1107 (-4.67\%). These results are in close conformite with the finding of Singh and Paroda (1989) ${ }^{[5]}$ and Sarode (1997) ${ }^{[4]}$ who also reported negative heterosis for days to maturity. Heterotic studied for number of pods per plant positive heterosis is also most important related to increase the yield. Out of 24 crosses 7, 2 crosses showed significant and positive average heterosis over mid parent and better parent respectively. The cross Chanoli x GAU-1107 depicted the highest significant positive average heterosis $(21.81 \%)$ and heterobeltiosis (12.89\%). The cross PDKV kanchan x GJG-0814 depicted the highest significant and positive standard heterosis over checks JAKI-9218 (13.20\%). For this character good amount of heterosis was reported by Tiwari and Pandey (1987) ${ }^{[6]}$, Singh and Paroda (1989) ${ }^{[5]}$. For 100 seed weight, Out of 24 crosses, 6, 6 crosses showed positive heterosis over mid parent and heterobeltiosis respectively. The cross Phule Vikram x C-1821 exhibited highest significant and positive average heterosis (13.36\%) and heterobeltiosis (13.84\%). The same cross exhibited significant and positive standard heterosis $23.07 \%$ over check JAKI-9218. Similar result was previously recorded by Gupta et al. (2003) ${ }^{[2]}$ and Tiwari and Pandey (1987) ${ }^{[6]}$. Seed yield is the complex character decides the economic worth of the crosses. Out of 24 crosses, 4, 1 crosses exhibited significant positive heterosis over mid parent and better parent respectively. The cross PDKV Kanchan $\mathrm{x}$ GJG-0814 exhibited highest significant and positive average heterosis $(25.68 \%)$ and heterosbeltiosis (20.22\%). The same cross exhibited positive standard heterosis $15.45 \%$ over check JAKI-9218. The significant positive heterosis for seed yield per plant has also been reported by Singh and Paroda (1989) ${ }^{[5]}$, Kulkarni et al. (2004) ${ }^{[3]}$ and Gadekar and Dodiya (2013) [1].

Table 1: Analysis of variance for various traits in line $\mathrm{x}$ tester analysis of chickpea

\begin{tabular}{|c|c|c|c|c|c|c|c|c|c|c|c|c|}
\hline Sou & d.f. & $\begin{array}{l}\text { Plant } \\
\text { stand }\end{array}$ & $\begin{array}{c}\text { Days to } \\
50 \% \\
\text { flowering }\end{array}$ & $\begin{array}{l}\text { Days to } \\
\text { Maturity }\end{array}$ & $\begin{array}{c}\text { Plant } \\
\text { Height } \\
(\mathbf{c m})\end{array}$ & \begin{tabular}{|c|} 
No. of \\
Primary \\
branches per \\
plant
\end{tabular} & $\begin{array}{c}\text { No. of } \\
\text { Secondary } \\
\text { branches per } \\
\text { plant } \\
\end{array}$ & $\begin{array}{c}\text { No. of } \\
\text { pods per } \\
\text { plant }\end{array}$ & $\begin{array}{c}100 \text { seed } \\
\text { weight per } \\
\text { plant }(g)\end{array}$ & \begin{tabular}{|c} 
Seed \\
yield per \\
plant $(\mathrm{g})$
\end{tabular} & $\begin{array}{c}\text { No. of } \\
\text { Seeds per } \\
\text { pod }\end{array}$ & \begin{tabular}{|c|} 
No. of \\
empty \\
pods per \\
plant
\end{tabular} \\
\hline & & 1 & 2 & 3 & 4 & 5 & 6 & 7 & $\overline{8}$ & 9 & 10 & 11 \\
\hline $\mathrm{Re}$ & 1 & .941 & & 85 & 11.97 & .64 & 22.36 & & & & & 0.024 \\
\hline Trea & 33 & $3.09 * *$ & $34.53^{* *}$ & $8.690 * *$ & $56.52 * *$ & $0.738^{* *}$ & $18.58 *$ & $* *$ & $.77 * *$ & $67.67 * *$ & $.014 * *$ & $2.19 * *$ \\
\hline Par & 9 & & & & 52.89 ** & 0.312 & 3044 & & & & & $3.11^{* *}$ \\
\hline $\begin{array}{r}\text { Paren } \\
\text { Cros }\end{array}$ & 1 & $20.88 * *$ & 8.24 & $67.81 * *$ & 1.067 & $9.531 * *$ & 11.96 & $390.72 * *$ & $20 *$ & 6.504 & 008 & $20.31 * *$ \\
\hline & 2 & $*$ & & & $60.35 * *$ & $0.522 * *$ & $24.59 * *$ & & & & & 1.04 \\
\hline Error & 33 & 0.729 & 13.72 & 2.515 & 9.873 & 0.177 & 9.464 & 28.76 & 2.497 & 4.951 & 0.006 & 0.700 \\
\hline
\end{tabular}

Note: * Significant at $5 \%$ level of significance, ** Significant at $1 \%$ level of significance 
Table 2: Heterosis (\%) over mid-parent (MP), better-parent (BP) and standard checks for different characters in chickpea

\begin{tabular}{|c|c|c|c|c|c|c|c|c|c|c|c|c|c|c|c|c|}
\hline \multirow{2}{*}{$\begin{array}{l}\text { Sr. } \\
\text { No. }\end{array}$} & \multirow[b]{2}{*}{ Crosses } & \multicolumn{3}{|c|}{ Plant stand } & \multicolumn{3}{|c|}{ Days to $50 \%$ Flowering } & \multicolumn{3}{|c|}{ Days to maturity } & \multicolumn{3}{|c|}{ Plant Height } & \multicolumn{3}{|c|}{ No. of Primary Branches } \\
\hline & & $\mathbf{M P}\left(\mathbf{H}_{1}\right)$ & $\mathbf{B P}\left(\mathbf{H}_{2}\right)$ & $\mathbf{S C}\left(\mathbf{H}_{3}\right)$ & $\mathbf{M P}\left(\mathbf{H}_{1}\right)$ & $\mathbf{B P}\left(\mathbf{H}_{2}\right)$ & $\mathrm{SC}\left(\mathrm{H}_{3}\right)$ & $\mathbf{M P}\left(\mathbf{H}_{1}\right)$ & $\mathbf{B P}\left(\mathbf{H}_{2}\right)$ & $\mathrm{SC}\left(\mathrm{H}_{3}\right)$ & $\mathbf{M P}\left(\mathbf{H}_{1}\right)$ & $\mathbf{B P}\left(\mathrm{H}_{2}\right)$ & $\mathbf{S C}\left(\mathrm{H}_{3}\right)$ & $\mathbf{M P}\left(\mathbf{H}_{1}\right)$ & $\mathbf{B P}\left(\mathbf{H}_{2}\right)$ & $\mathbf{S C}\left(\mathbf{H}_{3}\right)$ \\
\hline 1 & PDKVKanchan x GAU-1107 & $-24.37 * *$ & $-25.00 * *$ & $-25.00 * *$ & $-16.59^{*}$ & $-18.52^{*}$ & -8.33 & 1.2 & -1.87 & $3.96^{*}$ & 1.62 & -3.51 & -4.37 & $\mathrm{MP}\left(\mathrm{H}_{1}\right)$ & $\mathrm{BP}\left(\mathrm{H}_{2}\right)$ & $\mathrm{SC}\left(\mathrm{H}_{3}\right)$ \\
\hline 2 & PDKVKanchan x GJC-3 & $-26.67 * *$ & \begin{tabular}{|l|}
$-26.67 * *$ \\
\end{tabular} & $-26.67 * *$ & 7.62 & 4.63 & $17.71 *$ & 1.22 & -0.48 & 2.48 & 1.28 & -2.38 & -3.24 & $-31.75 * *$ & -35.51 & -30.00 \\
\hline 3 & PDKVKanchan x WR-315 & $-30.00 * *$ & $-30.00 * *$ & $-30.00 * *$ & -0.95 & -3.7 & 8.33 & $4.48^{* * *}$ & $4.48^{* *}$ & $3.96^{*}$ & 2.67 & -0.15 & -1.03 & -6.83 & -12.58 & -5.12 \\
\hline 4 & PDKVKanchan x C-1821 & $-28.33 * *$ & $-28.33 * *$ & $-28.33 * *$ & -2.39 & -5.56 & 6.25 & $3.65^{*}$ & 1.43 & $5.45 * *$ & -0.7 & -1.98 & -2.85 & $-16.14 *$ & $-20.00 * *$ & -13.17 \\
\hline 5 & PDKVKanchan x JCP-101 & $-8.33 * *$ & $-8.33 * *$ & $-8.33 * *$ & 7.84 & 1.85 & 14.58 & $2.96^{*}$ & 1.95 & $3.47 *$ & 7.84 & 6.29 & 5.35 & -7.11 & -8.99 & -1.22 \\
\hline 6 & PDKVKanchan x GJG-0814 & $-26.05 * *$ & \begin{tabular}{|l|}
$-26.67 * *$ \\
\end{tabular} & $-26.67 * *$ & -9.95 & -12.04 & -1.04 & $3.52 *$ & 2.49 & 1.98 & $15.90 * *$ & $14.21 *$ & $13.20^{*}$ & 4.9 & 3.37 & 12.2 \\
\hline 7 & PhuleVikram x GAU-1107 & $-26.05 * *$ & $-26.67 * *$ & $-26.67 * *$ & 9.27 & 8.74 & $16.67^{*}$ & $-3.09 *$ & $-4.67 * *$ & 0.99 & 4.91 & -0.74 & $\begin{array}{c}-0.88 \\
-1\end{array}$ & 9.05 & 6.97 & $16.10^{*}$ \\
\hline 8 & PhuleVikram x GJC-3 & $-11.67 * *$ & $-11.67 * *$ & $-11.67 * *$ & $-14.71 *$ & -14.71 & -9.38 & $3.61 *$ & $3.37 *$ & $6.44 * *$ & $11.79 *$ & 7.37 & 7.21 & -1.92 & -6.42 & -0.49 \\
\hline 9 & PhuleVikram x WR-315 & $-28.33 * *$ & $-28.33 * *$ & $-28.33 * *$ & 11.76 & 11.76 & $18.75^{*}$ & 1.47 & 0 & 2.48 & 4.82 & 1.57 & 1.42 & 10.65 & 4.82 & 11.46 \\
\hline 10 & PhuleVikram x C-1821 & $-25.00 * *$ & $-25.00 * *$ & $-25.00 * *$ & 1.01 & -1.96 & 4.17 & -0.49 & -0.97 & 1.49 & 9.33 & 7.37 & 7.21 & 5.48 & 1.61 & 8.05 \\
\hline 11 & PhuleVikram x JCP-101 & $-11.67 * *$ & $-11.67 * *$ & $-11.67 * *$ & -0.49 & -0.98 & 5.21 & 2.16 & 1.43 & $5.45 * *$ & -3.02 & -4.62 & -4.76 & $13.36^{*}$ & 12.84 & $20.00^{*}$ \\
\hline 12 & PhuleVikram x GJG-0814 & $-29.41 * *$ & $-30.00 * *$ & $-30.00 * *$ & 7.32 & 6.8 & 14.58 & $5.94 * *$ & $3.38^{*}$ & $5.94 * *$ & -4.8 & -6.54 & -6.67 & -6.84 & -7.8 & -1.95 \\
\hline 13 & AKG-1303 x GAU-1107 & $-27.73 * *$ & $-28.33 * *$ & $-28.33 * *$ & 3.48 & 0.97 & 8.33 & -0.47 & -1.87 & $3.96^{*}$ & -1.82 & -7.04 & -7.31 & 1.39 & 0.46 & 6.83 \\
\hline 14 & AKG-1303 x GJC-3 & $-26.67 * *$ & \begin{tabular}{|l|}
$-26.67 * *$ \\
\end{tabular} & $-26.67 * *$ & 3 & 0.98 & 7.29 & 1.44 & 1.44 & $4.46^{* * *}$ & -1.38 & -5.22 & -5.5 & 4.89 & $\begin{array}{l}-0.68 \\
-10\end{array}$ & 7.32 \\
\hline 15 & AKG-1303 x WR-315 & $-13.33 * *$ & \begin{tabular}{|l|}
$-13.33 * *$ \\
\end{tabular} & $-13.33 * *$ & 10 & 7.84 & 14.58 & $3.18^{*}$ & 1.44 & $4.46 * *$ & -1.34 & -4.33 & -4.61 & -2.52 & -8.35 & -0.98 \\
\hline 16 & AKG-1303 x C-1821 & $-15.00 * *$ & $-15.00 * *$ & $-15.00 * *$ & -2.06 & -3.06 & -1.04 & 1.69 & 0.96 & $3.96^{*}$ & -3.56 & -5.22 & -5.5 & -0.59 & -4.97 & 2.68 \\
\hline 17 & AKG-1303 x JCP-101 & $-28.33 * *$ & \begin{tabular}{|l|}
$-28.33 * * *$ \\
\end{tabular} & $-28.33 * *$ & 9.55 & 7.92 & 13.54 & 0 & -0.48 & $3.47 *$ & 9.2 & 7.48 & 7.16 & -4.91 & -6.09 & 1.46 \\
\hline 18 & AKG-1303 x GJG-0814 & $-22.69 * *$ & $-23.33 * *$ & $-23.33 * *$ & 1.49 & -0.97 & 6.25 & 1.73 & -0.96 & 1.98 & 4.88 & 3.05 & 2.75 & 8.51 & 6.55 & 15.12 \\
\hline 19 & Chanoli x GAU-1107 & $-25.86 * *$ & $-27.12 * *$ & $-28.33 * *$ & 11.01 & 5.22 & $26.04 * *$ & 1.9 & 0 & $5.94 * *$ & $21.81 * *$ & $12.89 *$ & 0.59 & -12.28 & -13.77 & -6.83 \\
\hline 20 & Chanoli x GJC-3 & $-29.91 * *$ & $-31.67 * *$ & $-31.67 * *$ & 9.68 & 3.48 & $23.96^{* * *}$ & $2.90^{*}$ & 2.4 & $5.45^{* * *}$ & $16.36^{* * *}$ & 6.3 & -2.26 & $-37.23 * *$ & $-55.30 * *$ & $-56.83^{* *}$ \\
\hline 21 & Chanoli x WR-315 & $-26.50 * *$ & \begin{tabular}{|l|}
$-28.33 * *$ \\
\end{tabular} & $-28.33 * *$ & -1.38 & -6.96 & 11.46 & 2.7 & 1.46 & $3.47 *$ & $12.17 *$ & 1.62 & -4.81 & $-38.71 * *$ & $-56.15 * *$ & $-58.29 * *$ \\
\hline 22 & Chanoli x C-1821 & $-29.91 * *$ & $-31.67 * *$ & $-31.67 * *$ & 2.37 & -6.09 & 12.5 & $3.65^{*}$ & $3.40^{*}$ & $5.45^{* *}$ & 0.97 & -9.63 & $-13.00 *$ & $-42.31 * *$ & $-59.16 * *$ & $-59.76 * *$ \\
\hline 23 & Chanoli x JCP-101 & $-26.50 * *$ & $-28.33 * *$ & $-28.33 * *$ & 10.19 & 3.48 & $23.96 * *$ & 0.96 & 0 & $3.96 *$ & $17.57 * *$ & 5.08 & 1.47 & $-40.67 * *$ & $-58.80 * *$ & $-56.59 * *$ \\
\hline 24 & Chanoli x GJG-0814 & $-27.59 * *$ & $-28.81 * *$ & $-30.00 * *$ & 8.26 & 2.61 & $22.92 * *$ & $5.71 * *$ & $3.40^{*}$ & $5.45 * *$ & $12.33^{*}$ & 0.56 & -3.24 & $-35.80 * *$ & $-55.27 * *$ & $-53.41 * *$ \\
\hline & RANGE & -30 to- 8.33 & \begin{tabular}{|c|}
-31.67 to- \\
8.33
\end{tabular} & $\begin{array}{c}-31.67 \text { to- } \\
8.33\end{array}$ & $\begin{array}{c}-16.59 \text { to } \\
11.76\end{array}$ & $\begin{array}{c}-18.52 \text { to } \\
11.76\end{array}$ & $\begin{array}{c}-9.38 \text { to } \\
26.04\end{array}$ & $\begin{array}{c}-3.09 \text { to } \\
5.94\end{array}$ & \begin{tabular}{|c|}
-4.67 \\
to 4.48 \\
\end{tabular} & $\begin{array}{c}0.99 \text { to } \\
6.44\end{array}$ & $\begin{array}{c}-4.8 \text { to } \\
21.81\end{array}$ & $\begin{array}{c}-9.63 \text { to } \\
14.21\end{array}$ & $\begin{array}{l}-13 \text { to } \\
13.20\end{array}$ & $-43.29 * *$ & $-60.51 * *$ & $-58.78 * *$ \\
\hline & $\mathrm{SE}(\mathrm{D}) \pm$ & 0.74 & 0.85 & 0.85 & 3.21 & 3.70 & 3.70 & 1.37 & 1.59 & 1.59 & 4.64 & 5.36 & 36 & $\begin{array}{c}-43.29 \text { to } \\
13.36\end{array}$ & $\begin{array}{c}-60.51 \text { to } \\
12.84\end{array}$ & $\begin{array}{c}-59.76 \text { to } \\
20\end{array}$ \\
\hline & CD5\% & 1.53 & 1.77 & 1.77 & 6.64 & 7.66 & 7.66 & 2.84 & 3.28 & 3.28 & 9.61 & 11.09 & 11.09 & 1.37 & 1.58 & 1.58 \\
\hline & CD1\% & 2.07 & 2.40 & 2.40 & 9.01 & 10.40 & 10.40 & 3.86 & 4.45 & 4.45 & & & & 2.83 & 3.27 & 3.27 \\
\hline
\end{tabular}

Note :* Significant at 5\% level of significance, ** Significant at $1 \%$ level of significance

\begin{tabular}{|c|c|c|c|c|c|c|c|c|c|c|c|c|c|c|c|c|}
\hline \multirow{2}{*}{ Sr. No. } & \multirow{2}{*}{ Crosses } & \multicolumn{3}{|c|}{ No. of Secondary Branches } & \multicolumn{3}{|c|}{ No. of pods per plant } & \multicolumn{3}{|c|}{100 seed yield $(\mathrm{gm})$} & \multicolumn{4}{|c|}{ Seed yield per plant (g) } & \multicolumn{2}{|c|}{ No. of seeds per pod } \\
\hline & & $\mathbf{M P}\left(\mathbf{H}_{1}\right)$ & $\mathbf{B P}\left(\mathbf{H}_{2}\right)$ & $\mathrm{SC}\left(\mathrm{H}_{3}\right)$ & $\mathbf{M P}\left(\mathbf{H}_{1}\right)$ & $\mathbf{B P}\left(\mathbf{H}_{2}\right)$ & $\mathbf{S C}\left(\mathbf{H}_{3}\right)$ & $\mathbf{M P}\left(\mathbf{H}_{1}\right)$ & $\mathbf{B P}\left(\mathbf{H}_{2}\right)$ & $\mathrm{SC}\left(\mathrm{H}_{3}\right)$ & $\mathbf{M P}\left(\mathrm{H}_{1}\right)$ & $\mathbf{B P}\left(\mathbf{H}_{2}\right)$ & $\mathrm{SC}\left(\mathrm{H}_{3}\right)$ & $\mathbf{M P}\left(\mathbf{H}_{1}\right)$ & $\mathbf{B P}\left(\mathbf{H}_{2}\right)$ & $\mathrm{SC}\left(\mathrm{H}_{3}\right)$ \\
\hline 1 & PDKVKanchan x GAU-1107 & -13.91 & $-28.04 * *$ & $-30.90 * *$ & 1.62 & -3.51 & -4.37 & $-31.75 * *$ & -35.51 & -30.00 & -13.91 & $-28.04 * *$ & $-30.90 * *$ & -6.98 & -13.04 & -13.04 \\
\hline 2 & PDKVKanchan x GJC-3 & -2.22 & -9.13 & -12.73 & 1.28 & -2.38 & -3.24 & -6.83 & -12.58 & -5.12 & -2.22 & -9.13 & -12.73 & -11.11 & -13.04 & -13.04 \\
\hline 3 & PDKVKanchan x WR-315 & 5.85 & -3.7 & -7.52 & 2.67 & -0.15 & -1.03 & \begin{tabular}{|l|l|}
$-16.14 *$ \\
\end{tabular} & $-20.00 * *$ & -13.17 & 5.85 & -3.7 & -7.52 & -6.98 & -13.04 & -13.04 \\
\hline 4 & PDKVKanchan x C-1821 & -5.12 & -9.35 & -12.94 & -0.7 & -1.98 & -2.85 & -7.11 & -8.99 & -1.22 & -5.12 & -9.35 & -12.94 & 2.33 & -4.35 & -4.35 \\
\hline 5 & PDKVKanchan x JCP-101 & 16.91 & 11.96 & 7.52 & 7.84 & 6.29 & 5.35 & 4.9 & 3.37 & 12.2 & 16.91 & 11.96 & 7.52 & 2.13 & 0 & 4.35 \\
\hline 6 & PDKVKanchan x GJG-0814 & $25.68 * *$ & $20.22 *$ & 15.45 & $15.90^{* * *}$ & $14.21^{*}$ & $13.20^{*}$ & 9.05 & 6.97 & $16.10^{*}$ & $25.68 * *$ & $20.22 *$ & 15.45 & 2.33 & -4.35 & -4.35 \\
\hline 7 & PhuleVikram x GAU-1107 & 13.46 & -4.23 & -10.23 & 4.91 & -0.74 & -0.88 & -1.92 & -6.42 & -0.49 & 13.46 & -4.23 & -10.23 & 2.22 & -8 & 0 \\
\hline 8 & PhuleVikram x GJC-3 & $23.08^{*}$ & 15.68 & 8.43 & 11.79* & 7.37 & 7.21 & 10.65 & 4.82 & 11.46 & $23.08^{*}$ & 15.68 & 8.43 & 6.38 & 0 & 8.7 \\
\hline 9 & PhuleVikram x WR-315 & 10.56 & 1.69 & -4.68 & 4.82 & 1.57 & 1.42 & 5.48 & 1.61 & 8.05 & 10.56 & 1.69 & -4.68 & -11.11 & $-20.00 * *$ & -13.04 \\
\hline 10 & PhuleVikram x C-1821 & $21.84 *$ & 18.04 & 10.65 & 9.33 & 7.37 & 7.21 & $13.36^{*}$ & 12.84 & $20.00^{*}$ & $21.84 *$ & 18.04 & 10.65 & -6.12 & -8 & 0 \\
\hline 11 & PhuleVikram x JCP-101 & -12.67 & -15.59 & $-20.88 *$ & -3.02 & -4.62 & -4.76 & -6.84 & -7.8 & -1.95 & -12.67 & -15.59 & $-20.88^{*}$ & -11.11 & $-20.00 * *$ & -13.04 \\
\hline
\end{tabular}




\begin{tabular}{|c|c|c|c|c|c|c|c|c|c|c|c|c|c|c|c|c|}
\hline 12 & PhuleVikram x GJG-0814 & -7.34 & -10.33 & -15.95 & -4.8 & -6.54 & -6.67 & 1.39 & 0.46 & 6.83 & -7.34 & -10.33 & -15.95 & -2.22 & -12 & -4.35 \\
\hline 13 & AKG-1303 x GAU-1107 & 4.27 & -13.15 & -15.87 & -1.82 & -7.04 & -7.31 & 4.89 & -0.68 & 7.32 & 4.27 & -13.15 & -15.87 & -6.98 & -13.04 & -13.04 \\
\hline 14 & AKG-1303 x GJC-3 & -5.47 & -12.5 & -15.24 & -1.38 & -5.22 & -5.5 & -2.52 & -8.35 & -0.98 & -5.47 & -12.5 & -15.24 & -6.67 & -8.7 & -8.7 \\
\hline 15 & AKG-1303 x WR-315 & -5.07 & -13.97 & -16.66 & -1.34 & -4.33 & -4.61 & -0.59 & -4.97 & 2.68 & -5.07 & -13.97 & -16.66 & -6.98 & -13.04 & -13.04 \\
\hline 16 & AKG-1303 x C-1821 & -13.22 & -17.24 & $-19.83^{*}$ & -3.56 & -5.22 & -5.5 & -4.91 & -6.09 & 1.46 & -13.22 & -17.24 & $-19.83 *$ & $-14.89^{*}$ & $-16.67^{*}$ & -13.04 \\
\hline 17 & AKG-1303 x JCP-101 & $19.37 *$ & 13.58 & 10.02 & 9.2 & 7.48 & 7.16 & 8.51 & 6.55 & 15.12 & $19.37 *$ & 13.58 & 10.02 & 6.98 & 0 & 0 \\
\hline 18 & AKG-1303 x GJG-0814 & -8.39 & -12.74 & -15.47 & 4.88 & 3.05 & 2.75 & -12.28 & -13.77 & -6.83 & -8.39 & -12.74 & -15.47 & -6.98 & -13.04 & -13.04 \\
\hline 19 & Chanoli x GAU-1107 & -18.33 & $-36.57 *$ & $-59.08 * *$ & $21.81 * *$ & $12.89 *$ & 0.59 & $-37.23 * *$ & $-55.30 * *$ & $-56.83 * *$ & -18.33 & $-36.57 *$ & $-59.08 * *$ & 0 & 0 & -13.04 \\
\hline 20 & Chanoli x GJC-3 & $-39.61 * *$ & $-56.73 * *$ & $-64.32 * *$ & $16.36^{* * *}$ & 6.3 & -2.26 & $-38.71 * *$ & $-56.15 * *$ & $-58.29 * *$ & $-39.61 * *$ & $-56.73 * *$ & $-64.32 * *$ & -4.76 & -9.09 & -13.04 \\
\hline 21 & Chanoli x WR-315 & $-38.69 *$ & $-55.44 * *$ & $-64.93 * *$ & $12.17 *$ & 1.62 & -4.81 & $-42.31 * *$ & $-59.16 * *$ & $-59.76 * *$ & $\begin{array}{l}-38.69 * \\
\end{array}$ & $-55.44 * *$ & $-64.93 * *$ & $20.00 * *$ & $20.00 *$ & 4.35 \\
\hline 22 & Chanoli x C-1821 & $-45.74 * *$ & $-61.85^{* *}$ & $-66.47 * *$ & 0.97 & -9.63 & $-13.00 *$ & $-40.67 * *$ & $\mid-58.80 * *$ & $-56.59 * *$ & $-45.74 * *$ & $-61.85^{* *}$ & $-66.47 * *$ & -9.09 & $-16.67 *$ & -13.04 \\
\hline 23 & Chanoli x JCP-101 & -26.1 & $-47.97^{* *}$ & $-54.49 * *$ & $17.57 * *$ & 5.08 & 1.47 & $-35.80 * *$ & $-55.27 * *$ & $-53.41 * *$ & -26.1 & $-47.97 * *$ & $-54.49 * *$ & 0 & 0 & -13.04 \\
\hline 24 & Chanoli x GJG-0814 & $-43.82 * *$ & $-60.48 * *$ & $-65.34 * *$ & $12.33 *$ & 0.56 & -3.24 & $-43.29 * *$ & $-60.51 * *$ & $-58.78 * *$ & $-43.82 * *$ & $-60.48 * *$ & $-65.34 * *$ & 0 & 0 & -13.04 \\
\hline & RANGE & -45.74 to 25.68 & -61.85 to 20.22 & -66.47 to 15.45 & $\begin{array}{c}-4.8 \text { to } \\
21.81\end{array}$ & $\begin{array}{c}-9.63 \text { to } \\
14.21\end{array}$ & \begin{tabular}{l|}
-13 to \\
13.20
\end{tabular} & \begin{tabular}{|c|}
-43.29 to \\
13.36 \\
\end{tabular} & \begin{tabular}{|c|}
-60.51 to \\
12.84 \\
\end{tabular} & $\begin{array}{c}-59.76 \text { to } \\
20 \\
\end{array}$ & $\begin{array}{c}-45.74 t \\
25.68\end{array}$ & $\begin{array}{c}-61.85 \text { to } \\
20.22\end{array}$ & $\begin{array}{c}-66.47 \text { to } \\
15.45\end{array}$ & -14.89 to 20 & -20.00 to 20.00 & -13.04 to 4.35 \\
\hline & $\mathrm{SE}(\mathrm{D}) \pm$ & 1.93 & 2.22 & 2.22 & 4.64 & 5.36 & 5.36 & 1.37 & 1.58 & 1.58 & 1.93 & 2.22 & 2.22 & 0.07 & 0.08 & 0.08 \\
\hline & CD5\% & 3.99 & 4.60 & 4.60 & 9.61 & 11.09 & 11.09 & 2.83 & 3.27 & 3.27 & 3.99 & 4.60 & 4.60 & 0.14 & 0.16 & 0.16 \\
\hline & CD1\% & 5.41 & 6.25 & 6.25 & 13.04 & 15.05 & 15.05 & 3.84 & 4.44 & 4.44 & 5.41 & 6.25 & 6.25 & 0.19 & 0.22 & 0.22 \\
\hline
\end{tabular}

Note :* Significant at $5 \%$ level of significance, ** Significant at $1 \%$ level of significance

\begin{tabular}{|c|c|c|c|c|}
\hline \multirow{2}{*}{ Sr. No. } & \multirow{2}{*}{ Crosses } & \multicolumn{3}{|c|}{ No. of Empty pods per plant } \\
\cline { 3 - 5 } & & $\mathbf{M P}\left(\mathbf{H}_{\mathbf{1}}\right)$ & $\mathbf{B P}\left(\mathbf{H}_{\mathbf{2}}\right)$ & $\mathbf{S C}\left(\mathbf{H}_{\mathbf{3}}\right)$ \\
\hline 1 & PDKVKanchan x GAU-1107 & $320.00^{*}$ & 110 & 10.53 \\
\hline 2 & PDKVKanchan x GJC-3 & 100 & 0 & 5.26 \\
\hline 3 & PDKVKanchan x WR-315 & 211.1 & 55.56 & -26.3 \\
\hline 4 & PDKVKanchan x C-1821 & 0 & 0 & -21.1 \\
\hline 5 & PDKVKanchan x JCP-101 & 0 & 0 & 5.26 \\
\hline 6 & PDKVKanchan x GJG-0814 & 69.49 & -15.3 & 31.58 \\
\hline 7 & PhuleVikram x GAU-1107 & $300.00^{*}$ & 100 & 5.26 \\
\hline 8 & PhuleVikram x GJC-3 & $200.00^{*}$ & 50 & 57.89 \\
\hline 9 & PhuleVikram x WR-315 & $466.67^{* *}$ & 183.3 & 34.21 \\
\hline 10 & PhuleVikram x C-1821 & 0 & 0 & -2.63 \\
\hline 11 & PhuleVikram x JCP-101 & 0 & 0 & 0 \\
\hline 12 & PhuleVikram x GJG-0814 & 8.47 & -45.8 & -15.8 \\
\hline 13 & AKG-1303 x GAU-1107 & $670.00^{* *}$ & $285.00^{* *}$ & $102.63^{*}$ \\
\hline 14 & AKG-1303 x GJC-3 & $200.00^{*}$ & 50 & 57.89 \\
\hline 15 & AKG-1303 x WR-315 & $566.67^{* *}$ & $233.33^{*}$ & 57.89 \\
\hline 16 & AKG-1303 x C-1821 & 0 & 0 & -21.1 \\
\hline 17 & AKG-1303 x JCP-101 & 0 & 0 & 52.63 \\
\hline 18 & AKG-1303 x GJG-0814 & $103.39^{*}$ & 1.69 & 57.89 \\
\hline 19 & Chanoli x GAU-1107 & -6.17 & -37.7 & 0 \\
\hline 20 & Chanoli x GJC-3 & 22.77 & 1.64 & 63.16 \\
\hline 21 & Chanoli x WR-315 & -44.3 & $-63.93^{*}$ & -42.1 \\
\hline 22 & Chanoli x C-1821 & -27.9 & $-63.93^{*}$ & -42.1 \\
\hline 23 & Chanoli x JCP-101 & 4.92 & -47.5 & -15.8 \\
\hline 24 & Chanoli x GJG-0814 & -30 & -31.2 & 10.53 \\
\hline & RANGE & -44.3 to 670 & -63.93 to 285 & -42.1 to102.63 \\
\hline & SE(D) & 0.72 & 0.84 & 0.84 \\
\hline & CD5\% & 1.5 & 1.73 & 1.73 \\
\hline & CD1\% & 2.03 & 2.35 & 2.35 \\
\hline & & & \\
\hline
\end{tabular}

$\sim 1622 \sim$ 


\section{Conclusion}

In this study, line $\mathrm{x}$ tester analysis on the basis of mean seed yield performance, average heterosis, heterobeltiosis, standard heterosis, the crosses PDKV Kanchan x GJG-0814, AKG$1303 \times$ JCP-101 and Phule Vikram x JGC-3 were found to be the most promising crosses among all the 24 crosses studied. And the cross Phule Vikram $\times$ GJC-3 recorded early for $50 \%$ flowering and maturity.

\section{References}

1. Gadekar MS, Dodiya NS. Heterosis and combining ability analysis for yield \& yield contributing traits in chickpea, Legume research 2013;36(5):373-379.

2. Gupta S, Sarvjeet S, Ajindev K. Heterosis for seed yield and its component traits in desi x desi and desi x Kabuli crosses of chickpea (Cicer arietinum. L.). Crop Improvement 2003;30:143-149.

3. Kulkarni SS, Patil JV, Gawande VL. Heterosis studies in chickpea (Cicer arietinum L.) Journal Maharashtra Agric. Univ 2004;29(3):272-276.

4. Sarode ND, Deshmukh RB, Kute NS, Kanawade DG, Dhone SR. Genetic analysis in chickpea. Legume Res 1997;24(3):159-163.

5. Singh O, Paroda RS. A comparative analysis combining ability of heterosis in irradiated and non- irradiated diallel populations of chickpea. Indian J Pulses Res 1989;2(1):19.

6. Tiwari SK, Pandey MP. Heterosis an inbreeding depression in chickpea. Indian $\mathrm{J}$ Genet 1987;47(3):261264.

7. Panse VG, Sukhatme PV. Statistical methods for agricultural workers, ICAR, New Delhi, India 1958. 